\title{
Searches for Muon to Electron Conversion
}

\author{
Richard Bonventre ${ }^{* \dagger}$ \\ Lawrence Berkeley National Lab \\ E-mail: rbonventre@lbl.gov
}

The Mu2e experiment at Fermilab and the COMET experiment at J-PARC will search for the conversion of muons to electrons in the field of a nucleus. Muon to electron conversion and other charged lepton flavor violating processes can be sensitive probes of physics beyond the standard model. These experiments will provide a four orders of magnitude improvement over current limits, with single event sensitivities of nearly $10^{-17}$. The experimental design and status of these experiments is discussed.

Flavor Physics and CP Violation,

6-9 June 2016

Caltech, Pasadena CA, USA

${ }^{*}$ Speaker.

${ }^{\dagger}$ Member of the Mu2e Collaboration 


\section{Introduction}

Charged lepton flavor violation (CLFV) can occur in the Standard Model through loops involving neutrinos. However small neutrino masses leads to these processes being highly suppressed; for example, the branching ratio of $\mu \rightarrow e \gamma$ is predicted to be less than $10^{-54}$. This ratio is for all intents and purposes unobservable, and so any observation of CLFV would be unambiguous evidence of physics beyond the Standard Model. Many models of new physics, including supersymmetric models, leptoquarks, models with second Higgs doublets, etc., predict additional contributions to CLFV, with branching ratios of up to $10^{-15}[1,2,3]$.

Currently the most restrictive CLFV measurements are from MEG, with a limit of $\mathscr{B}(\mu \rightarrow$ $e \gamma)<4.2 \times 10^{-13}$, and SINDRUM-II with a limit of $R_{\mu e}<6 \times 10^{-13}$ for muon conversion with a gold target $[5,6]$. One can compare how strongly new physics can be constrained by $\mu \rightarrow e \gamma$ and $\mu N \rightarrow e N$ measurements using a model independent framework. Generically, we expect new contributions to CLFV to come from physics at some large mass scale $\Lambda$. We can write an effective Lagrangian,

$$
\mathscr{L}_{C L F V}=\frac{m_{\mu}}{(1+\kappa) \Lambda^{2}} \bar{\mu}_{R} \sigma_{\mu v} e_{L} F^{\mu v}+\frac{\kappa}{(1+\kappa) \Lambda^{2}} \bar{\mu}_{L} \gamma_{\mu} e_{L}\left(\sum_{q=u, d} \bar{q}_{L} \gamma^{\mu} q_{L}\right)
$$

with the parameter $\kappa$ determining the relative strength of "loop" terms and "contact" terms [4]. The expected mass scale reach for a given branching ratio limit is shown in Fig. 1. Both $\mu \rightarrow e \gamma$ and $\mu N \rightarrow e N$ measurements are sensitive to models where loop terms dominate $(\kappa \ll 1)$, but only $\mu N \rightarrow e N$ is sensitive when contact terms do $(\kappa \gg 1)$. In addition, at the expected sensitivity of Mu2e and COMET of $10^{-17}$, effective mass scales up to $10^{4} \mathrm{TeV}$ are being probed, beyond the range of collider experiments.

Muon conversion experiments stop slow muons in a target where they form muonic atoms, quickly dropping to the lowest energy 1s state. After some delay the muons decay, convert, or are captured by the nucleus. For negative muons, decay or conversion of the bound muon happens coherently. In the case of a neutrinoless conversion a monoenergetic electron with an energy of about $105 \mathrm{MeV}$ is produced. On the other hand, muon decay in orbit (DIO) will produce both electrons and neutrinos, but again the coherent recoil off the nucleus allows the Michel energy spectrum endpoint to be pushed all the way up to the conversion peak. A precise energy measurement is required to separate the high energy DIO tail from the peak at $105 \mathrm{MeV}$ that would be a signal of CLFV.

Reaching a sensitivity of $10^{-17}$ also requires stopping at least $10^{17}$ muons. There are many additional backgrounds associated with muon production and capture, for example, gammas produced from radiative pion capture on the stopping target. This makes it difficult to achieve these statistics while maintaining a clean measurement. Both Mu2e and COMET use pulsed beams and take advantage of the lifetime of the muonic atom by using a delayed signal window to greatly suppress beam related backgrounds.

The parameter measured by these experiments is the rate of muon to electron conversion normalized to the rate of muon capture by the nucleus:

$$
R_{\mu e}=\frac{\mu^{-}+A(Z, N) \rightarrow e^{-}+A(Z, N)}{\mu^{-}+A(Z, N) \rightarrow v_{\mu}+A(Z-1, N)} .
$$




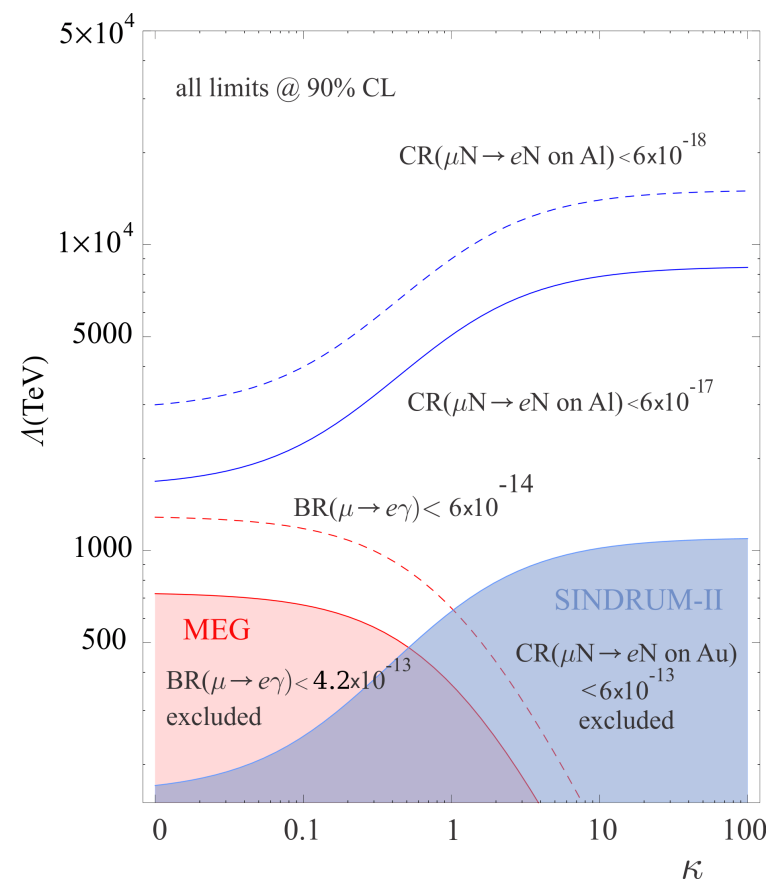

Figure 1: Current limits for MEG (filled red) and SINDRUM-II (filled blue) compared to predicted sensitivies of MEG-II (dashed red), Mu2e and COMET (blue), and a possible future Mu2e upgrade (dashed blue). $\Lambda$ is the effective mass scale of new physics assuming unit coupling and $\kappa$ parameterizes the relative contributions of "loop" and "contact" terms [7]. MEG result from [5].

\section{Mu2e experiment}

Mu2e uses an $8 \mathrm{GeV}$ pulsed proton beam from the Fermilab booster. The protons are produced in bunches separated by $1.7 \mu \mathrm{s}$, with a ratio of in-time to out-of-time protons of less than $10^{-10}$. The protons hit a tungsten target inside the Mu2e experiment, producing pions and eventually muons. The beam bunching and out of time proton extinction are used to create a time window clean of beam related backgrounds, as shown in Fig. 2.

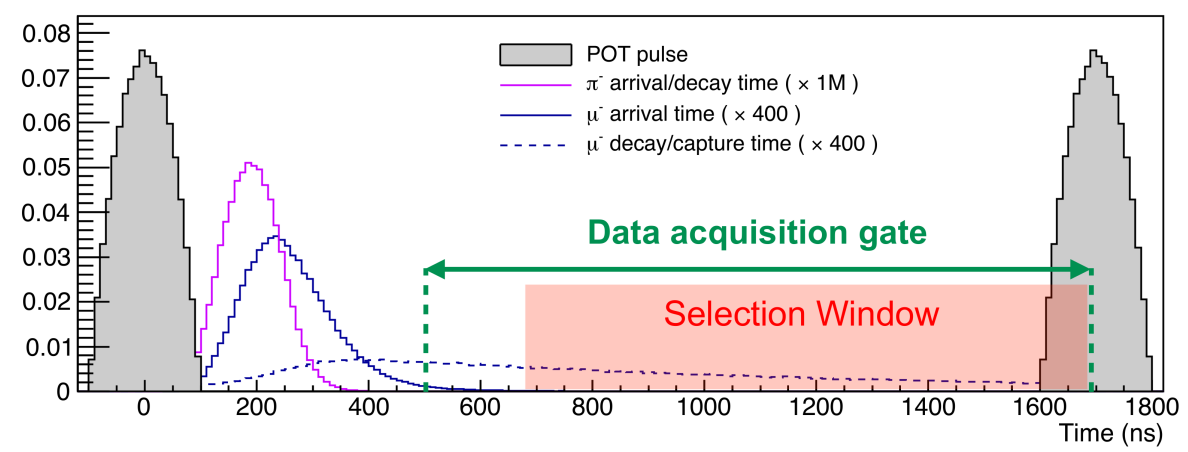

Figure 2: Mu2e delayed signal window compared to the time structure of signal events and backgrounds. 
The layout of the Mu2e experiment is shown in Fig. 3. It consists of three regions of superconducting solenoids: the production solenoid (PS), transport solenoid (TS), and detector solenoid (TS). The tungsten production target is located in a gradient field in the DS, which reflects low momentum pions and muons back towards the TS, increasing collection efficiency. The TS is an $\mathrm{S}$-shaped solenoid that separates particles in the beam by charge and momentum. Collimators are used to select for low momentum muons. The DS contains the stopping target, made of thin foils of aluminum, followed by a straw tracker and calorimeter. The stopping target also sits in a gradient field which reflects conversion electrons towards the detector elements, again increasing the efficiency.

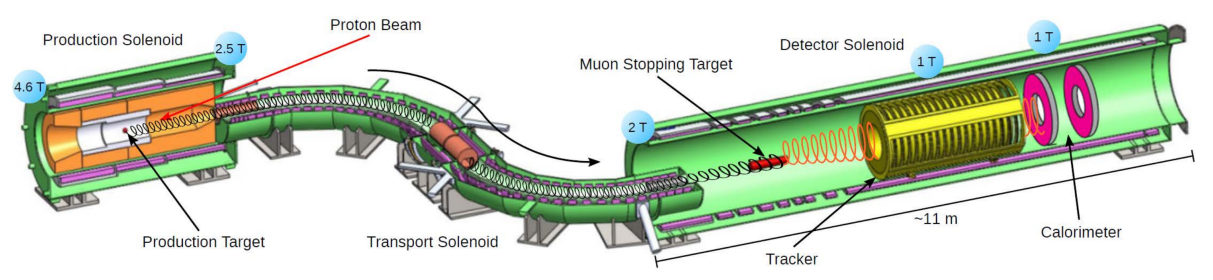

Figure 3: Layout of the Mu2e experiment.

The main detector elements of Mu2e are a straw tracker and a calorimeter. The tracker is made of low-mass straws in vacuum, and sits in a constant $1 \mathrm{~T}$ field. It consists of 20,000 straws each $5 \mathrm{~mm}$ in diameter and made of $15 \mu \mathrm{m}$ thick mylar. The straws are perpendicular to the beam, and oriented such that they do not pass within a minimum radius, allowing the bulk of the DIO electrons and other beam backgrounds to pass through without interacting with the tracker. The expected momentum resolution is better than $200 \mathrm{keV} / \mathrm{c}$. Fig. 4 shows the reconstructed momentum of conversion electrons compared to the DIO background from a full simulation of the Mu2e detector.

The electromagnetic calorimeter is located downstream of the tracker. It consists of two annular disks each containing 678 CsI scintillating crystals read out by SiPMs. It is designed to have a $5 \%$ energy resolution and a $0.5 \mathrm{~ns}$ timing resolution independent of the tracker. This allows it to perform particle identification which is required to reject muon tracks. It additionally can provide a seed for the track reconstruction.

The DS and parts of the TS are also covered by a cosmic ray veto (CRV). Cosmic rays can produce tracks indistinguishable from conversion electrons by delta ray production or muon decay, and so an active veto is necessary. The CRV consists of four overlapping layers of scintillator read out on both ends by SiPMs. It will reject cosmic rays with better than $10^{-4}$ inefficiency.

Overall fewer than 0.5 background events are expected over Mu2e's entire running time. In a 3 year run there will be $3.6 \times 10^{20}$ protons on target leading to about $10^{18}$ stopped muons and a single event sensitivity of $R_{\mu e}=2.4 \times 10^{-17}$.

\section{COMET experiment}

The COMET experiment has the same fundamental design as Mu2e; an $8 \mathrm{GeV}$ pulsed proton 


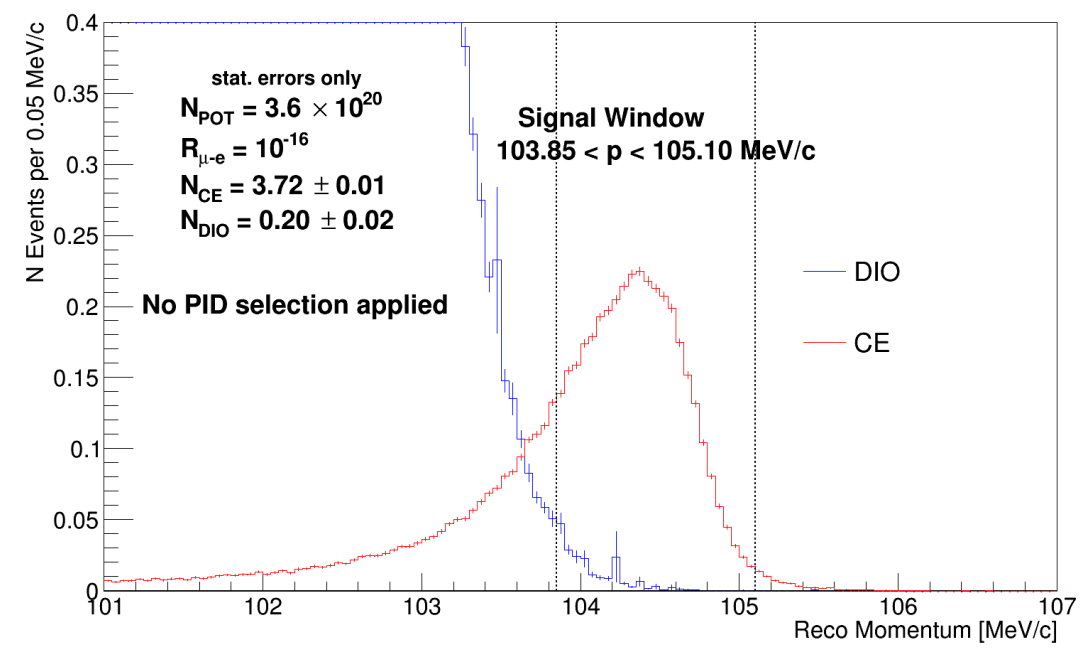

Figure 4: Reconstructed momentum distribution for DIO (blue) and conversion (red) electrons from Mu2e simulation of a three year run. Signal size assumes $R_{\mu e}=10^{-16}$.

beam is used to create slow muons which are stopped in an aluminum target, and the momentum of produced particles is measured in a straw tracker. The main difference is in the design of the muon transport and the layout of the detectors. COMET is also planned to be run in two phases, with an early phase designed to measure backgrounds for the future full scale design. An overview of the design of each phase is shown in Fig. 5.
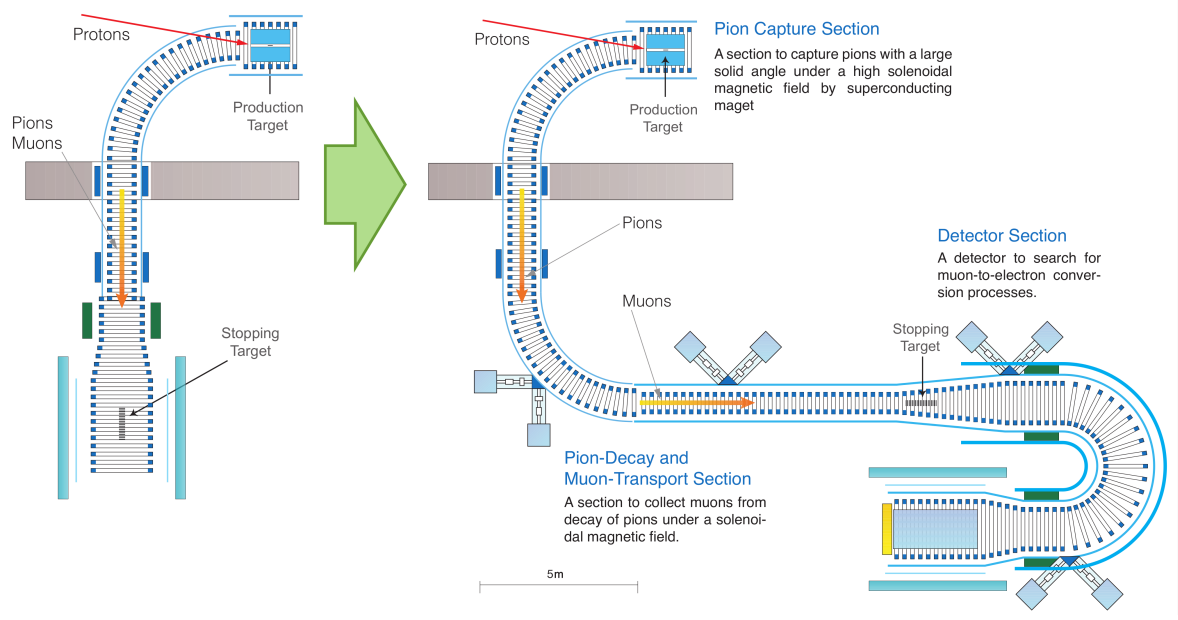

Figure 5: Layout of COMET Phase-I (left) and Phase-II (right).

As in Mu2e, COMET uses a bent transport solenoid to prevent particles besides low momentum muons produced from the proton beam and the production target from reaching the stopping target. In Phase-II, a C-shaped transport solenoid is used instead of the S-shaped solenoid used 
in Mu2e. In addition, the stopping target is then followed by another $\mathrm{C}$-shaped solenoid section, which allows for the removal of a large fraction of the DIO electrons as well as muon capture products before they can reach the detector elements. This allows the COMET straw tracker to instrument all radii.

For Phase-I, the transport solenoid is reduced down to a single $90^{\circ}$ turn. Additionally, there is no second bend after the stopping target to remove backgrounds. For this reason in this phase the straw tracker will be only used to study backgrounds. A cylindrical drift chamber surrounding the stopping target will be used for a physics measurement. After a 110 day run Phase-I will reach a S.E.S. of $R_{\mu e}=3.1 \times 10^{-15}$. The upgraded transport and detector design in addition to a higher intensity beam for Phase-II will increase that sensitivity to $3 \times 10^{-17}$.

\section{Current Status}

The various elements of the Mu2e design have gone through extensive testing. TS modules prototypes have been produced and tested, and contracts have been awarded for construction of the PS and DS. A calorimeter prototype module was tested in an electron beam and shown to have sufficient energy and time resolution. Several straw tracker prototypes have been built, both to test mechanical construction as well as to measure performance with cosmic rays and proton beam tests. Time resolution, efficiency, and cross talk were measured and found to meet specifications. A functional full scale preproduction prototype of a 96 straw panel is currently being developed. Additionally, a CRV prototype was tested in the Fermilab proton test beam. The Mu2e experiment has now received full CD-3 approval and is preparing to enter full scale production. The detector hall and beamline construction is well under way. Construction is expected to be completed in 2021 , followed by a 3 year physics run.

Civil construction as well as production of the detectors for COMET Phase-I is also currently under way. The TS has been completed, and wire stringing for the cylindrical drift chamber is currently in progress. Phase-I data taking is scheduled to begin in 2018, followed by construction of Phase-II, which is scheduled to begin running in 2021.

\section{Summary}

Mu2e and COMET plan to look for CLFV by measuring the rate of coherent neutrinoless muon to electron conversion using muons stopped in an aluminum target. By using intense pulsed proton beams these experiments can overcome the backgrounds limiting previous measurements, and plan to improve current limits by four orders of magnitude. Both experiments plan to start taking data in 2021, with a single event sensitivity of about $10^{-17}$.

\section{Acknowledgements}

I would like to thank Yoshitaka Kuno and Hisataka Yoshida for providing information on the COMET experiment.

I am grateful for the vital contributions of the Fermilab staff and the technical staff of the participating institutions. My work on Mu2e is supported by the US Department of Energy; the 
Italian Istituto Nazionale di Fisica Nucleare; the US National Science Foundation; the Ministry of Education and Science of the Russian Federation; the Thousand Talents Plan of China; the Helmholtz Association of Germany; and the EU Horizon 2020 Research and Innovation Program under the Marie Sklodowska-Curie Grant Agreement No.690385. Fermilab is operated by Fermi Research Alliance, LLC under Contract No. De-AC02-07CH11359 with the US Department of Energy.

\section{References}

[1] Y. Kuno, Y. Okada, Muon decay and physics beyond the standard model, Rev. Mod. Phys. 73, 151 (2001).

[2] W.J. Marciano, T. Mori, J.M. Roney, Charged Lepton Flavor Violation Experiments, Ann. Rev. Nucl. Part. Sci. 58, 315 (2008).

[3] M. Raidal et al, Flavour physics of leptons and dipole moments, Eur. Phys. J. C 57 13, (2008).

[4] A. de Gouvea, P. Vogel, Lepton flavor and number conservation, and physics beyond the standard model, Prog. Part. Nucl. Phys. 7175 (2013).

[5] A. M. Baldini et al. (MEG Collaboration), Search for the Lepton Flavour Violating Decay $\mu^{+} \rightarrow e^{+} \gamma$ with the Full Dataset of the MEG Experiment, arXiv:1605.05081 (2016).

[6] W. Bertl et al. (SINDRUM-II Collaboration), A search for $\mu$-e conversion in muonic gold, Eur. Phys. J. C 47, 337 (2006).

[7] A. de Gouvea, (Charged) Lepton Flavor Violation, Nucl. Phys. B (Proc. Suppl.) 188, 303 (2009). 